\title{
RICCI-FLAT DEFORMATIONS OF METRICS WITH EXCEPTIONAL HOLONOMY
}

\author{
JOHANNES NORDSTRÖM
}

\begin{abstract}
Let $G$ be one of the Ricci-flat holonomy groups $S U(n), \operatorname{Sp}(n), \operatorname{Spin}(7)$ or $G_{2}$, and $M$ a compact manifold of dimension $2 n, 4 n, 8$ or 7 , respectively. We prove that the natural map from the moduli space of torsion-free $G$-structures on $M$ to the moduli space of Ricci-flat metrics is open, and that the image is a smooth manifold. For the exceptional cases $G=\operatorname{Spin}(7)$ and $G_{2}$ we extend the result to asymptotically cylindrical manifolds.
\end{abstract}

\section{INTRODUCTION}

The possible holonomy groups of simply-connected non-symmetric irreducible Riemannian manifolds were classified by Berger [1. 'Berger's list' contains several infinite families, and the two exceptional cases $\operatorname{Spin}(7)$ and $G_{2}$, appearing as the holonomy of manifolds of dimension 8 and 7 respectively. In many cases, an effective approach to studying $G$-metrics (by which we mean metrics with holonomy contained in $G$ ) is to define them in terms of certain closed differential forms, equivalent to torsion-free $G$-structures. A $G$-structure defines a Riemannian metric, and if its torsion vanishes (which is a first-order differential equation) then the induced metric has holonomy contained in $G$. For $G=S U(n), S p(n), \operatorname{Spin}(7)$ or $G_{2}$ we define a $G$-manifold to be a connected oriented manifold of dimension $2 n, 4 n, 8$ or 7 respectively, equipped with a torsion-free $G$-structure and the associated Riemannian metric.

$G$-metrics are Ricci-flat for $G=S U(n), S p(n), S p i n(7)$ or $G_{2}$. For compact manifolds M.Y. Wang [22, Theorem 3.1] proved a local converse: any small Ricci-flat deformation of a $G$-metric still has holonomy contained in $G$. In other words, the moduli space $\mathcal{W}_{G}$ of $G$-metrics is an open subset of the moduli space $\mathcal{W}_{0}$ of Ricci-flat metrics. This is an analogue of a result of Koiso [10] on deformations of Kähler-Einstein metrics. Wang proves the result case by case, but asks if there is a general proof.

In this paper we observe that the problem can be reduced in a uniform way to showing unobstructedness for deformations of torsion-free $G$-structures. This has in turn been given a uniform treatment by Goto [7. As part of the proof we provide a clear summary of the deformation theory of Ricci-flat metrics on a compact manifold (a special case of deformation theory for Einstein metrics used by Koiso [10]). This treatment makes it easier to extend the results to other types of complete manifolds, and we will discuss the asymptotically cylindrical case in some detail.

If $M$ is a compact $G$-manifold then the group $\mathcal{D}$ of diffeomorphisms of $M$ isotopic to the identity acts on the space of torsion-free $G$-structures by pull-backs. The resulting quotient is the moduli space $\mathcal{M}_{G}$ of torsion-free $G$-structures on $M$, and is known to be a manifold. This is due to Tian [19] and Todorov [20] in the Calabi-Yau $(G=S U(n))$ case, and Joyce in the exceptional cases (see [9, $§ 10.4,10.7]) . \mathcal{D}$ also acts on the space of Riemannian metrics, and we let $\mathcal{W}_{G}$ and $\mathcal{W}_{0}$ denote the moduli spaces of $G$-metrics and Ricci-flat metrics respectively. In $\$ 3$ we prove

Theorem I. Let $G=S U(n), S p(n)$, Spin $(7)$ or $G_{2}$, and let $M$ be a compact $G$-manifold. Then $\mathcal{W}_{G}$ is open in $\mathcal{W}_{0}$. Moreover, $\mathcal{W}_{G}$ is a smooth manifold and the natural map

$$
m: \mathcal{M}_{G} \rightarrow \mathcal{W}_{G}
$$

that sends a torsion-free $G$-structure to the metric it defines is a submersion. 
Remark 1.1. It is easy to see that $\mathcal{W}_{G}$ is also closed in $\mathcal{W}_{0}$, so it is a union of connected components. It seems to be an open problem whether there exist any compact Ricci-flat manifolds without a holonomy reduction.

Remark 1.2. The quotient of the space of $G$-metrics by the group of all diffeomorphisms of $M$ (not just the ones isotopic to the identity) is a quotient of $\mathcal{W}_{G}$ with discrete fibres and in general an orbifold (cf. remark 3.11).

The case $G=G_{2}$ of theorem I was proved by M.Y. Wang [22, Theorem 3.1B]. For $G=S p(n)$ or $\operatorname{Spin}(7)$, Wang showed that $\mathcal{W}_{G} \subseteq \mathcal{W}_{0}$ is open (so the statement of theorem I is stronger). Manifolds with holonomy in $S U(n)$ are Calabi-Yau manifolds, i.e. Ricci-flat Kähler manifolds. The case $G=S U(n)$ of theorem I is therefore a special case of a more general result by Koiso on Einstein deformations of Kähler-Einstein metrics.

Let $X^{2 n}$ be a compact Kähler-Einstein manifold. Koiso [10, Theorem 0.7] shows that if the Einstein constant $e$ (equivalently the first Chern class $c_{1}(X)$ ) is non-positive and the complex deformations of $X$ are unobstructed, then any small Einstein deformation of the metric is Kähler with respect to some perturbed complex structure. In other words, the map from the moduli space of Kähler-Einstein structures to the moduli space of Einstein metrics is open (see e.g. [2, §12J] for a discussion). The proof shows that near any Kähler-Einstein metric there is a smooth pre-moduli space of Einstein metrics, so that the moduli space of Kähler-Einstein metrics is an orbifold. Tian [19 and Todorov 20] show that on a compact Calabi-Yau manifold the obstructions to the complex deformations vanish. Hence theorem I for $G=S U(n)$ follows from Koiso's theorem, except for the claim that $\mathcal{W}_{S U(n)}$ is smooth (and not just an orbifold).

Remark 1.3. Dai, X. Wang and Wei [5] use the fact that $\mathcal{W}_{G}$ is open in $\mathcal{W}$ to deduce that any scalar-flat deformation of a Ricci-flat $G$-metric on a compact manifold remains a $G$-metric.

The proof of theorem I given in 93 is a simplification of Wang's argument for the case $G=G_{2}$. First, we observe that the point-wise surjectivity of the derivative of $m$ follows from a well-known property of Laplacians on manifolds with reduced holonomy noted by Chern [4]. This makes it easy to see that the proof applies also for the other Ricci-flat holonomy groups, provided that the deformations of torsion-free $G$-structures are unobstructed. Second, we streamline some parts of the deformation theory for Ricci-flat metrics. This makes it easier to generalise the result to certain non-compact settings.

One relevant type of complete non-compact Riemannian manifolds are exponentially asymptotically cylindrical (EAC) ones (defined in 92.7 ). In 3.5 we explain that the necessary deformation theory for Ricci-flat metrics and (at least when $G=\operatorname{Spin}(7)$ or $G_{2}$ ) torsion-free $G$-structures carries over to the EAC case, so that there are smooth moduli spaces $\mathcal{M}_{G}$ and $\mathcal{W}_{0}$ of torsion-free EAC $G$-structures and Ricci-flat EAC metrics on an EAC $G$-manifold $M$.

Theorem I'. Let $G=\operatorname{Spin}(7)$ or $G_{2}$, and $M$ an EAC G-manifold. Then $\mathcal{W}_{G}$ is open in $\mathcal{W}_{0}$. Moreover, $\mathcal{W}_{G}$ is a smooth manifold and the natural map

$$
m: \mathcal{M}_{G} \rightarrow \mathcal{W}_{G}
$$

is a submersion.

In [1] Kovalev proves the analogous result for EAC Calabi-Yau manifolds, by an extension of Koiso's arguments for the compact Kähler-Einstein case. The discussion in subsection 3.5 of deformations of EAC Ricci-flat metrics is similar to that in [11, while the necessary results on deformations of EAC $G$-structures are taken from [17].

Remark 1.4. One may consider the structure of the map $m$ in greater detail. For the exceptional cases $G=G_{2}$ and $\operatorname{Spin}(7)$, one can use the characterisation of torsion-free $G$-structures in terms of parallel spinors (cf. M.Y. Wang [21]) to show that $m$ is a diffeomorphism if the holonomy of $M$ is exactly $G$ for any $G$-metric (this depends only on the topology of $M$ ), and that $\mathcal{M}_{G}$ is in general a disjoint union of fibre bundles over $\mathcal{W}_{G}$ with real projective plane fibres (the components corrspond to different spin structures on $M$ ). See [16, §5.3] for details. For Calabi-Yau manifolds, [2, Theorem 12.103] states that the moduli space of Calabi-Yau structures on a compact manifold 
is a locally trivial fibration with compact fibres over the moduli space of Calabi-Yau metrics (but does not describe the fibres further).

Acknowledgements. I am grateful to Alexei Kovalev for many helpful discussions.

\section{Preliminaries}

We describe how a metric with holonomy $G=\operatorname{Spin}(7), G_{2}, S U(n)$ or $S p(n)$ can be defined in terms of a torsion-free $G$-structure. This is a set of differential forms, that are both parallel and harmonic. For more background on manifolds with special holonomy see e.g. Joyce [9] or Salamon [18.

2.1. Holonomy. We define the holonomy group of a Riemannian manifold. For a fuller discussion of holonomy see e.g. [9, Chapter 2].

Definition 2.1. Let $M^{n}$ be a manifold with a Riemannian metric $g$. If $x \in M$ and $\gamma$ is a closed piecewise $C^{1}$ loop in $M$ based at $x$ then the parallel transport around $\gamma$ (with respect to the Levi-Civita connection) defines an orthogonal linear map $P_{\gamma}: T_{x} M \rightarrow T_{x} M$. The holonomy group $\operatorname{Hol}(g, x) \subseteq O\left(T_{x} M\right)$ at $x$ is the group generated by $\left\{P_{\gamma}: \gamma\right.$ is a closed loop based at $\left.x\right\}$.

If $x, y \in M$ and $\tau$ is a path from $x$ to $y$ we can define a group isomorphism $H o l(g, x) \rightarrow H o l(g, y)$ by $P_{\gamma} \mapsto P_{\tau} \circ P_{\gamma} \circ P_{\tau}^{-1}$. Provided that $M$ is connected we can therefore identify $\operatorname{Hol}(g, x)$ with a subgroup of $O(n)$, independently of $x$ up to conjugacy, and talk simply of the holonomy group of $g$.

There is a correspondence between tensors fixed by the holonomy group and parallel tensor fields on the manifold.

Proposition 2.2 ([9, Proposition 2.5.2]). Let $M^{n}$ be a Riemannian manifold, $x \in M$ and $E$ a vector bundle on $M$ associated to TM. If $s$ is a parallel section of $E$ then $s(x)$ is preserved by $\mathrm{Hol}(g, x)$. Conversely if $s_{0} \in E_{x}$ is preserved by $\operatorname{Hol}(g, x)$ then there is a parallel section $s$ of $E$ such that $s(x)=s_{0}$.

Definition 2.3. Let $M^{n}$ a manifold and $G \subseteq O(n)$ a closed subgroup. A $G$-metric on $M$ is a metric with holonomy contained in $G$.

2.2. $\operatorname{Spin}(7)$-structures. The stabiliser in $G L\left(\mathbb{R}^{8}\right)$ of

$$
\begin{aligned}
\psi_{0}=d x^{1234}+d x^{1256}+d x^{1278}+d x^{1357}-d x^{1368}-d x^{1458}-d x^{1467}- \\
d x^{2358}-d x^{2367}-d x^{2457}+d x^{2468}+d x^{3456}+d x^{3478}+d x^{5678} \in \Lambda^{4}\left(\mathbb{R}^{8}\right)^{*}
\end{aligned}
$$

is $\operatorname{Spin}(7)$ (identified with a subgroup of $S O(8)$ by the spin representation). For an oriented vector space $V$ of dimension 8 let $\Lambda_{\operatorname{Spin}(7)} V^{*} \subset \Lambda^{4} V^{*}$ be the subset of forms equivalent to $\psi_{0}$ under some oriented linear isomorphism $V \cong \mathbb{R}^{8}$. A $\operatorname{Spin}(7)$-structure on an oriented manifold $M^{8}$ is a section of the subbundle $\Lambda_{\operatorname{Spin}(7)} T^{*} M \subset \Lambda^{4} T^{*} M$. Since $\operatorname{Spin}(7) \subset S O(8)$ a $\operatorname{Spin}(7)$-structure $\psi$ naturally defines a Riemannian metric $g_{\psi}$ on $M$. Note that $\psi$ is self-dual with respect to this metric.

We make a note of the decomposition of $\Lambda^{4} \mathbb{R}^{8}$ into irreducible representations of $\operatorname{Spin}(7)$. Firstly it splits into the self-dual and anti-self-dual parts $\Lambda_{ \pm}^{4} \mathbb{R}^{8}$. We let $\Lambda_{d}^{4} \mathbb{R}^{8}$ denote an irreducible component of rank $d$. Then

$$
\begin{gathered}
\Lambda_{+}^{4} \mathbb{R}^{8}=\Lambda_{1}^{4} \mathbb{R}^{8} \oplus \Lambda_{7}^{4} \mathbb{R}^{8} \oplus \Lambda_{27}^{4} \mathbb{R}^{8}, \\
\Lambda_{-}^{4} \mathbb{R}^{8}=\Lambda_{35}^{4} \mathbb{R}^{8} .
\end{gathered}
$$

The tangent space at $\psi$ to the space of $\operatorname{Spin}(7)$-structures $\Gamma\left(\Lambda_{\operatorname{Spin}(7)} T^{*} M\right)$ is $\Gamma\left(E_{\psi}\right)$, where $E_{\psi} \subset \Lambda^{4} T^{*} M$ is a $\operatorname{Spin}(7)$-invariant linear subbundle. More precisely, the $\operatorname{Spin}(7)$-structure $\psi$ determines a decomposition of $\Lambda^{4} T^{*} M$ modelled on (2), and $E_{\psi}=\Lambda_{1 \oplus 7 \oplus 35}^{4} T^{*} M$.

A $\operatorname{Spin}(7)$-structure $\psi$ is torsion-free if it is parallel with respect to the metric it induces. It follows immediately from proposition 2.2 that a metric $g$ on $M^{8}$ has holonomy contained in $\operatorname{Spin}(7)$ if and only if it is induced by a torsion-free $\operatorname{Spin}(7)$-structures. 
The condition that $\operatorname{Hol}(g) \subseteq \operatorname{Spin}(7)$ imposes algebraic constraints on the curvature of $g$. In particular any Spin(7)-metric is Ricci-flat (see [18, Corollary 12.6]). The torsion-free condition for $\psi$ can usefully be rewritten as $d \psi=0$ (see [18, Lemma 12.4]).

2.3. $G_{2}$-structures. Recall that $G_{2}$ can be defined as the automorphism group of the normed algebra of octonions. Equivalently, $G_{2}$ is the stabiliser in $G L\left(\mathbb{R}^{7}\right)$ of

$$
\varphi_{0}=d x^{123}+d x^{145}+d x^{167}+d x^{246}-d x^{257}-d x^{347}-d x^{356} \in \Lambda^{3}\left(\mathbb{R}^{7}\right)^{*} .
$$

For an oriented vector space $V$ of dimension 7 let $\Lambda_{G_{2}} V^{*} \subset \Lambda^{3} V^{*}$ be the subset consisting of forms equivalent to $\varphi_{0}$ under some oriented linear isomorphism $V \cong \mathbb{R}^{7}$. A $G_{2}$-structure on an oriented manifold $M^{7}$ is a section $\varphi$ of the subbundle $\Lambda_{G_{2}} T^{*} M \subset \Lambda^{3} T^{*} M$, and naturally defines a Riemannian metric $g_{\varphi}$ on $M$.

The typical fibre of $\Lambda_{G_{2}} T^{*} M$ is isomorphic to $G L\left(\mathbb{R}^{7}\right) / G_{2}$, so by dimension-counting $\Lambda_{G_{2}} T^{*} M$ is an open subbundle of $\Lambda^{3} T^{*} M$. Thus the tangent space at $\varphi$ to the space of $G_{2}$-structures $\Gamma\left(\Lambda_{G_{2}} T^{*} M\right)$ is $\Omega^{3}(M)=\Gamma\left(E_{\varphi}\right)$, if we let $E_{\varphi}=\Lambda^{3} T^{*} M$.

A $G_{2}$-structure $\varphi$ is torsion-free if it is parallel with respect to the metric it induces. A metric $g$ on $M^{7}$ has holonomy contained in $G_{2}$ if and only if it is induced by a torsion-free $G_{2}$-structure.

$G_{2}$-metrics are Ricci-flat (see [18, Proposition 11.8]). As observed by Gray, a $G_{2}$-structure $\varphi$ is torsion-free if and only if $d \varphi=0$ and $d_{\varphi}^{*} \varphi=0$ (where the codifferential $d_{\varphi}^{*}$ is defined using the metric induced by $\varphi$, see [18, Lemma 11.5]).

2.4. $S U(n)$-structures. Let $z^{k}=x^{2 k-1}+i x^{2 k}$ be complex coordinates on $\mathbb{R}^{2 n}$. Then the stabiliser in $G L\left(\mathbb{R}^{2 n}\right)$ of the pair of forms

$$
\begin{gathered}
\Omega_{0}=d z^{1} \wedge \cdots \wedge d z^{n} \in \Lambda^{n}\left(\mathbb{R}^{2 n}\right)^{*} \otimes \mathbb{C} \\
\omega_{0}=\frac{i}{2}\left(d z^{1} \wedge d \bar{z}^{1}+\cdots d z^{n} \wedge d \bar{z}^{n}\right) \in \Lambda^{2}\left(\mathbb{R}^{2 n}\right)^{*}
\end{gathered}
$$

is $S U(n)$. For an oriented real vector space $V$ of dimension $2 n$ let $\Lambda_{S U(n)} V^{*} \subset \Lambda^{n} V_{\mathbb{C}}^{*} \oplus \Lambda^{2} V^{*}$ be the subset of pairs $(\Omega, \omega)$ equivalent to $\left(\Omega_{0}, \omega_{0}\right)$ under some oriented isomorphism $V \cong \mathbb{R}^{2 n}$. An $S U(n)$-structure on an oriented manifold $M^{2 n}$ is a section $(\Omega, \omega)$ of the subbundle $\Lambda_{S U(n)} T^{*} M \subset$ $\Lambda^{n} T_{\mathbb{C}}^{*} M \oplus \Lambda^{2} T^{*} M$. It naturally defines an almost complex structure and a Riemannian metric on $M$, such that $\Omega$ has type $(n, 0)$. The volume form on $V$ is given by both $(-1)^{\frac{n(n-1)}{2}}\left(\frac{i}{2}\right)^{n} \Omega \wedge \bar{\Omega}$ and $\frac{1}{n !} \omega^{n}$ (cf. Hitchin [8, §2]).

An $S U(n)$-structure is torsion-free if it is parallel with respect to the metric it induces, and a metric on $M^{2 n}$ has holonomy contained in $S U(n)$ if and only if it is induced by a torsion-free $S U(n)$-structure.

$(\Omega, \omega)$ is torsion-free if and only if $d \Omega=d \omega=0$. Then the induced almost complex structure is integrable, the Riemannian metric is a Ricci-flat Kähler metric, and $\Omega$ is a holomorphic $(n, 0)$-form. $M^{2 n}$ equipped with a torsion-free $S U(n)$-structure is called an $S U(n)$-manifold or Calabi-Yau $n$-fold.

2.5. $S p(n)$-structures. Let $q^{k}=x^{4 k-3}+i x^{4 k-2}+j x^{4 k-1}+k x^{4 k}$ be quaternionic coordinates on $\mathbb{R}^{4 n}$. Then we may write

$$
d q^{1} \wedge d \bar{q}^{1}+\cdots+d q^{n} \wedge d \bar{q}^{n}=-2\left(i \omega_{0}^{I}+j \omega_{0}^{J}+k \omega_{0}^{K}\right)
$$

with $\omega_{0}^{I}, \omega_{0}^{J}, \omega_{0}^{K} \in \Lambda^{2}\left(\mathbb{R}^{4 n}\right)^{*}$. The stabiliser in $G L\left(\mathbb{R}^{4 n}\right)$ of this triple of 2-forms is $S p(n)$. For an oriented real vector space $V$ of dimension $4 n$ let $\Lambda_{S p(n)} V^{*} \subset\left(\Lambda^{2} V^{*}\right)^{\otimes 3}$ be the subset of triples $\left(\omega^{I}, \omega^{J}, \omega^{K}\right)$ equivalent to $\left(\omega_{0}^{I}, \omega_{0}^{J}, \omega_{0}^{K}\right)$ under some oriented isomorphism $V \cong \mathbb{R}^{4 n}$. An $S p(n)$ structure on an oriented manifold $M^{4 n}$ is a section of the subbundle $\Lambda_{S p(n)} T^{*} M \subset\left(\Lambda^{2} T^{*} M\right)^{\otimes 3}$. It is torsion-free if it is parallel with respect to the induced metric, and a metric on $M^{4 n}$ has holonomy contained in $S p(n)$ if and only if it is induced by a torsion-free $S p(n)$-structure.

Equivalently, $\left(\omega^{I}, \omega^{J}, \omega^{K}\right)$ is torsion-free if and only if $d \omega^{I}=d \omega^{J}=d \omega^{K}=0$. Then the metric of $M$ is Ricci-flat, and $M$ has a triple $I, J, K$ of anti-commuting integrable complex structures, such that $\omega^{I}$ is the Kähler form and $\omega^{J}+i \omega^{K}$ a holomorphic (2,0)-form with respect to $I$, etc. $M^{4 n}$ equipped with a torsion-free $S p(n)$-structure is called an $S p(n)$-manifold or hyperKähler manifold. 
2.6. Laplacians. For a Riemannian manifold with holonomy $H$ one can define a Lichnerowicz Laplacian on vector bundles associated to the $H$-structure. On differential forms this agrees with the usual Hodge Laplacian, as is explained in Besse [2, §1I]. This can be used to define decompositions of the spaces of harmonic forms analogous to the Kähler decomposition on a Kähler manifold, as observed by Chern 4 .

Suppose a Riemannian manifold $M^{n}$ has holonomy group $\operatorname{Hol}(M) \subseteq H$ (where $H$ is a closed subgroup of $O(n))$, and a corresponding $H$-structure. Let $\rho: H \rightarrow G L(E)$ be a representation of $H$, and $E_{\rho}$ the corresponding associated vector bundle. Let $\mathfrak{h}_{\text {ad }}$ be the vector bundle induced by the adjoint representation. $\mathfrak{h}_{\text {ad }}$ can be identified with a subbundle of $\Lambda^{2} T^{*} M$, and because $H o l(M) \subseteq H$ the Riemannian curvature tensor $R$ is a (symmetric) section of $\mathfrak{h}_{\text {ad }} \otimes \mathfrak{h}_{\text {ad }}$. We use the Lie algebra representation $D \rho: \mathfrak{h} \rightarrow \operatorname{End}(E)$ to define

$$
(D \rho)^{2}: \mathfrak{h} \otimes \mathfrak{h} \rightarrow \operatorname{End}(E), \quad a \otimes b \mapsto D \rho(a) \circ D \rho(b) .
$$

This induces a bundle map $\mathfrak{h}_{\text {ad }} \otimes \mathfrak{h}_{\text {ad }} \rightarrow \operatorname{End}\left(E_{\rho}\right)$. The symmetry of $R$ implies that $(D \rho)^{2}(R)$ is a self-adjoint section of $\operatorname{End}\left(E_{\rho}\right)$.

Definition 2.4. Let $M$ be a Riemannian manifold with $\operatorname{Hol}(M) \subseteq H$ and $\rho$ a representation of $H$. The Lichnerowicz Laplacian on the associated vector bundle $E_{\rho}$ is the elliptic formally self-adjoint operator

$$
\triangle_{\rho}=\nabla^{*} \nabla-2(D \rho)^{2}(R): \Gamma\left(E_{\rho}\right) \rightarrow \Gamma\left(E_{\rho}\right),
$$

where $\nabla$ is the connection on $E_{\rho}$ induced by the Levi-Civita connection on $M$.

Lemma 2.5. Let $M^{n}$ be a Riemannian manifold. The Lichnerowicz Laplacian corresponding to the standard representation of $O(n)$ on $\Lambda^{m}\left(\mathbb{R}^{n}\right)^{*}$ is the usual Hodge Laplacian $\triangle$ on $\Lambda^{m} T^{*} M$.

Proof. See [2, Equation (1.154)].

Lemma 2.6 (cf. [9, Theorem 3.5.3]). Let $M$ be a Riemannian manifold with Hol $(M) \subseteq H$ and $\phi: E \rightarrow F$ an equivariant map of $H$-representations $(E, \rho),(F, \sigma)$. $\phi$ induces a bundle map $E_{\rho} \rightarrow F_{\sigma}$, and the diagram below commutes.

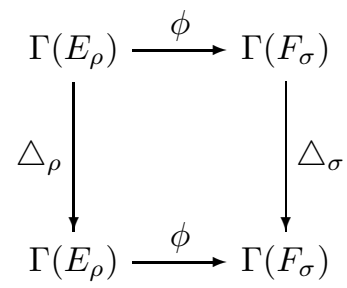

In particular, if $\rho_{1}, \rho_{2}$ are $H$-representations then $\triangle_{\rho_{1} \oplus \rho_{2}}=\triangle_{\rho_{1}} \oplus \triangle_{\rho_{2}}$.

Proof. Clear from the fact that the Lichnerowicz Laplacian is defined naturally by the representations.

Suppose that $\Lambda^{m}\left(\mathbb{R}^{n}\right)^{*}$ splits as a direct sum of representations of $H$. On a manifold $M$ with holonomy contained in $H$ there is a corresponding splitting of $\Lambda^{m} T^{*} M$ into $H$-invariant subbundles. Lemma 2.6 implies that the Hodge Laplacian commutes with the projections to the subbundles. Hence there is also a decomposition for the harmonic forms (see [9, Theorem 3.5.3]).

2.7. Asymptotically cylindrical manifolds. A non-compact manifold $M$ is said to have cylindrical ends if $M$ is written as union of two pieces $M_{0}$ and $M_{\infty}$ with common boundary $X$, where $M_{0}$ is compact, and $M_{\infty}$ is identified with $X \times \mathbb{R}^{+}$by a diffeomorphism (identifying $\partial M_{\infty}$ with $X \times\{0\}) . X$ is called the cross-section of $M$. Let $t$ be a smooth real function on $M$ which is the $\mathbb{R}^{+}$-coordinate on $M_{\infty}$, and negative on the interior of $M_{0}$. A tensor field $s$ on $M$ is said to be exponentially asymptotic with rate $\delta>0$ to a translation-invariant tensor $s_{\infty}$ on $M$ if $e^{\delta t}\left\|\nabla^{k}\left(s-s_{\infty}\right)\right\|$ is bounded on $M_{\infty}$ for all $k \geq 0$, with respect to a norm defined by an arbitrary Riemannian metric on $X$. 
A metric on $M$ is called $E A C$ (exponentially asymptotically cylindrical) if it is exponentially asymptotic to a product metric on $X \times \mathbb{R}$. Similarly a $G$-structure is said to be EAC if it is exponentially asymptotic to a translation-invariant $G$-structure on $X \times \mathbb{R}$ which defines a product metric. A diffeomorphism $\phi$ of $M$ is called EAC if it is exponentially close to a product diffeomorphism $(x, t) \mapsto(\Xi(x), t+h)$ of $X \times \mathbb{R}$ in a similar exponential sense.

Remark 2.7. If an EAC metric has reduced holonomy then so does the induced metric on the crosssection. In particular, the cross-section of an EAC Spin(7)-manifold is a compact $G_{2}$-manifold, and the cross-section of an EAC $G_{2}$-manifold is a Calabi-Yau 3-fold.

On an asymptotically cylindrical manifold $M$ it is useful to introduce weighted Hölder norms. Let $E$ be a vector bundle on $M$ associated to the tangent bundle, $k \geq 0, \alpha \in(0,1)$ and $\delta \in \mathbb{R}$. We define the $C_{\delta}^{k, \alpha}$-norm of a section $s$ of $E$ in terms of the usual Hölder norm by

$$
\|s\|_{C_{\delta}^{k, \alpha}}=\left\|e^{\delta t} s\right\|_{C^{k, \alpha}} .
$$

Denote the space of sections of $E$ with finite $C_{\delta}^{k, \alpha}$-norm by $C_{\delta}^{k, \alpha}(E)$. Up to Lipschitz equivalence the weighted norms are independent of the choice of asymptotically cylindrical metric, and of the choice of $t$ on the compact piece $M_{0}$. In particular, the topological vector spaces $C_{\delta}^{k, \alpha}(E)$ are independent of these choices.

The main importance of the weighted norms is that elliptic asymptotically translation-invariant operators acts as Fredholm operators on the weighted spaces of sections. In particular, this applies to the Hodge Laplacian of an EAC metric.

Theorem 2.8. Let $M$ be an asymptotically cylindrical manifold. If $\delta>0$ with $\delta^{2}$ smaller than any positive eigenvalue of the Laplacian on $X$ then

$$
\triangle: C_{ \pm \delta}^{k+2, \alpha}\left(\Lambda^{m} T^{*} M\right) \rightarrow C_{ \pm \delta}^{k, \alpha}\left(\Lambda^{m} T^{*} M\right)
$$

is Fredholm for all $m$. The index of (6) is $\mp\left(b^{m-1}(X)+b^{m}(X)\right)$.

Proof. The Fredholm result is a special case of Lockhart and McOwen [13, Theorem 6.2], while the index formula can be found in Lockhart [12, §3] (or Melrose [14, §6.4]).

This can be used to deduce results analogous to Hodge theory for compact manifolds. Let $\mathcal{H}_{0}^{m}$ denote the space of bounded harmonic $m$-forms on $M$, and $\mathcal{H}_{\infty}^{m}$ the translation-invariant harmonic forms on $X \times \mathbb{R}$. $\mathcal{H}_{\infty}^{m}=\mathcal{H}_{X}^{m} \oplus d t \wedge \mathcal{H}_{X}^{m-1}$, where $\mathcal{H}_{X}^{m}$ are the harmonic forms on $X$. Any $\phi \in \mathcal{H}_{0}^{m}$ is asymptotically translation-invariant; let $B(\phi) \in \mathcal{H}_{\infty}^{m}$ denote its limit. We can write $B(\phi)=B_{a}(\phi)+d t \wedge B_{e}(\phi) \in \mathcal{H}_{X}^{m} \oplus d t \wedge \mathcal{H}_{X}^{m-1}$. Then

$$
\mathcal{H}_{0}^{m}=\mathcal{H}_{a b s}^{m} \oplus \mathcal{H}_{E}^{m},
$$

where $\mathcal{H}_{a b s}^{m}$ is the kernel of $B_{e}: \mathcal{H}_{0}^{m} \rightarrow \mathcal{H}_{X}^{m-1}$, and $\mathcal{H}_{E}^{m} \subset \mathcal{H}_{0}^{m}$ is the subspace of exact forms.

Theorem 2.9. Let $M$ be an EAC manifold. The natural map $\mathcal{H}_{\text {abs }}^{m} \rightarrow H^{m}(M)$ is an isomorphism.

Dually $\mathcal{H}_{E}^{m}$ is isomorphic to the kernel of the homomorphism $e: H_{c p t}^{m}(M) \rightarrow H^{m}(M)$ induced by the natural chain inclusion $\Omega_{c p t}^{*}(M) \rightarrow \Omega^{*}(M)$. If $M$ has a single end (i.e. the cross-section $X$ is connected) then the long exact sequence for relative cohomology of $(M, X)$ shows that $e: H_{c p t}^{1}(M) \rightarrow H^{1}(M)$ is injective. Hence

Corollary 2.10. Let $M^{n}$ be an asymptotically cylindrical manifold which has a single end (i.e. the cross-section $X$ is connected). Then $\mathcal{H}_{E}^{1}=0$, and $\mathcal{H}_{0}^{1} \rightarrow H^{1}(M)$ is an isomorphism.

\section{RicCi-Flat DEFormations of $G$-MEtRiCS}

3.1. Deformations of $G$-metrics. Let $G$ be one of the Ricci-flat holonomy groups $S U(n), S p(n)$, $\operatorname{Spin}(7)$ or $G_{2}$, and $M$ a compact $G$-manifold. We explained in 92 how a $G$-metric on a manifold $M$ of the appropriate dimension can be defined in terms of a $G$-structure, i.e. a section of a subbundle $\Lambda_{G} T^{*} M \subset \Lambda^{*} T^{*} M$, which is torsion-free and in particular closed. In order to prove theorem I we will use that deformations of $G$-structures are unobstructed, and the existence of pre-moduli spaces. 
The tangent space to $\Gamma\left(\Lambda_{G} T^{*} M\right)$ at a $G$-structure $\chi$ consists of the sections of the bundle of point-wise tangents to $\Lambda_{G} T^{*} M$ at $\chi$, which is a vector bundle $E_{\chi} \subseteq \Lambda^{*} T^{*} M$ associated to the $G$-structure. $E_{\chi}$ is a bundle of forms, so the Hodge Laplacian acts on $\Gamma\left(E_{\chi}\right)$. When $\chi$ is torsion-free this is the same as the Lichnerowicz Laplacian from $\$ 2.6$

The group $\mathcal{D}$ of diffeomorphisms of $M$ isotopic to the identity acts on the space of torsion-free $G$-structures by pull-backs and the quotient is the moduli space $\mathcal{M}_{G}$ of torsion-free $G$-structures. Goto [7] proves that the deformations of torsion-free $G$-structures are unobstructed in the following sense:

Proposition 3.1. Let $G=S U(n), S p(n)$, Spin $(7)$ or $G_{2}, M$ a compact $G$-manifold, and $\chi$ a torsion-free $G$-structure on $M$. Then there is a submanifold $\mathcal{R}$ of the space of $C^{1} G$-structures such that

(i) the elements of $\mathcal{R}$ are smooth torsion-free $G$-structures,

(ii) the tangent space to $\mathcal{R}$ at $\chi$ is the space of harmonic sections of $E_{\chi}$,

(iii) the natural map $\mathcal{R} \rightarrow \mathcal{M}_{G}$ is a homeomorphism onto a neighbourhood of $\chi \mathcal{D}$ in $\mathcal{M}_{G}$.

The spaces $\mathcal{R}$ are pre-moduli spaces of torsion-free $G$-structures and can be used as coordinate charts for $\mathcal{M}_{G}$, which is thus a smooth manifold. The pre-moduli space $\mathcal{R}$ near $\chi$ can be chosen to be invariant under the stabiliser $\chi$. In fact

Proposition 3.2. Let $\chi \in \mathcal{X}$, and let $\mathcal{I}_{\chi} \subseteq \mathcal{D}$ be the stabiliser of $\chi$. If $\mathcal{R}$ is $\mathcal{I}_{\chi}$-invariant and small enough then $\mathcal{I}_{x}$ acts trivially on $\mathcal{R}$ and $\mathcal{I}_{\chi^{\prime}}=\mathcal{I}_{x}$ for all $\chi^{\prime} \in \mathcal{R}$.

Proof. Because the tangent space to $\mathcal{R}$ consists of harmonic forms, a neighbourhood of $\chi$ can be immersed in (a direct sum of copies of) the de Rham cohomology of $M$. Because elements of $\mathcal{I}_{\chi}$ act trivially on cohomology they must fix such a neighbourhood. The reverse inclusion $\mathcal{I}_{\chi^{\prime}} \subseteq \mathcal{I}_{\chi}$ follows from [6, Theorem 7.1(2)].

3.2. Killing vector fields. Before we discuss the deformations of Ricci-flat metrics we make some remarks about Killing vector fields. These are the infinitesimal isometries of a Riemannian manifold $(M, g)$, i.e. vector fields $V$ such that the Lie derivative $\mathcal{L}_{V} g$ vanishes.

Definition 3.3. Given a metric $g$ on $M$ let $\delta^{*}: \Omega^{1}(M) \rightarrow \Gamma\left(S^{2}\left(T^{*} M\right)\right)$ be the symmetric part of the Levi-Civita connection $\nabla: \Omega^{1}(M) \rightarrow \Gamma\left(T^{*} M \otimes T^{*} M\right)$.

The formal adjoint $\delta$ of $\delta^{*}$ is the restriction of $\nabla^{*}: \Gamma\left(T^{*} M \otimes T^{*} M\right) \rightarrow \Omega^{1}(M)$ to the symmetric part $\Gamma\left(S^{2}\left(T^{*} M\right)\right)$.

Proposition 3.4 ([2, Lemma 1.60]). Let $g$ be a Riemannian metric on a manifold $M$ and $V$ a vector field. Then $\mathcal{L}_{V} g=2 \delta^{*} V^{b}$, where $V^{b}$ denotes the 1 -form $g(V, \cdot)$.

The second Bianchi identity implies that

$$
(2 \delta+d \operatorname{tr}) R i c=0
$$

for any Riemannian metric. The operator $2 \delta+d$ tr is sometimes called the Bianchi operator, and it also satisfies the following useful identity.

Lemma 3.5 ([11, Equation (14)]). If $(M, g)$ is a Ricci-flat manifold then

$$
(2 \delta+d \operatorname{tr}) \delta^{*}=\triangle .
$$

Proof. The anti-symmetric part of $\nabla$ on $\Omega^{1}(M)$ is $\frac{1}{2} d$, so $\delta^{*}=\nabla-\frac{1}{2} d$. Also $\operatorname{tr} \delta^{*}=d^{*}$ on $\Omega^{1}(M)$. Using the Weitzenböck formula $\triangle=\nabla^{*} \nabla-$ Ric we obtain

$$
(2 \delta+d \operatorname{tr}) \delta^{*}=2 \nabla^{*} \nabla-\nabla^{*} d+d \operatorname{tr} \delta^{*}=2 \nabla^{*} \nabla-d^{*} d-d d^{*}=\triangle .
$$

Proposition 3.6. Let $(M, g)$ be a Ricci-flat manifold. If $V$ is a Killing field then the 1-form $V^{b}$ is harmonic. If $M$ is compact then the converse also holds.

Proof. $\delta^{*} V^{b}=0 \Rightarrow \triangle V^{b}=0$ by lemma 3.5. Trivially $\nabla V^{b}=0 \Rightarrow \delta^{*} V^{b}=0$, and if $M$ is compact then $\triangle V^{b}=\nabla^{*} \nabla V^{b}=0 \Rightarrow \nabla V^{b}=0$ by integration by parts.

This implies that, for any of the Ricci-flat holonomy groups $G$, the space of infinitesimal automorphisms of a compact $G$-manifold is $\left(\mathcal{H}^{1}\right)^{\sharp}$. 
3.3. Deformations of Ricci-flat metrics. We summarise some deformation theory for Ricciflat metrics. This is essentially taken from the explanation of the deformation theory for Einstein metrics in [2, $\S 12 \mathrm{C}]$ (in turn based on Koiso [10]), specialised to the Ricci-flat case. The main difference in this presentation is a slightly simplified technique in the 'slice argument'.

Let $M^{n}$ be a compact manifold. The diffeomorphism group $\mathcal{D}$ acts on the space of Ricci-flat metrics on $M$ by pull-backs. We define the moduli space $\mathcal{W}_{0}$ of Ricci-flat metrics to be the quotient of the space of Ricci-flat metrics by $\mathcal{D}$. (We do not divide by the rescaling action of $\mathbb{R}^{+}$too, as is done in [2].)

Take $k \geq 2$, and let $g$ be a Ricci-flat Riemannian metric on $M$. In order to study a neighbourhood of $g \mathcal{D}$ in $\mathcal{W}_{0}$ we use the usual technique of considering a transverse slice for the diffeomorphism action. Such a slice argument is explained very carefully in Ebin [6. In the current setting it is, however, possible to use elliptic regularity to avoid some of the technical subtleties of Ebin's argument. As in [17, §6.7], where a similar simplification is used, one advantage compared with Ebin's approach is that it is easier to extend to the asymptotically cylindrical case.

We include the space of smooth Riemannian metrics in the Hölder space $C^{k, \alpha}\left(S^{2} T^{*} M\right)$, and let $\mathcal{D}_{k+1}$ be the $C^{k+1, \alpha}$ completion of $\mathcal{D}\left(\mathcal{D}_{k+1}\right.$ is generated by exp of $C^{k+1, \alpha}$ vector fields). By proposition 3.4 the tangent space to the $\mathcal{D}_{k+1}$-orbit at $g$ is $\delta_{g}^{*} C^{k+1, \alpha}\left(\Lambda^{1}\right)$. Let $K$ be the kernel of $2 \delta_{g}+d \operatorname{tr}_{g}$ in $C^{k, \alpha}\left(S^{2} T^{*} M\right)$. Because $g$ is Ricci-flat, harmonic 1-forms are parallel and therefore $L^{2}$-orthogonal to the image of $2 \delta_{g}+d \operatorname{tr}_{g}$. It follows from lemma 3.5 and the Fredholm alternative for $\triangle_{g}$ on $\Omega^{1}(M)$ that there is a direct sum decomposition

$$
C^{k, \alpha}\left(S^{2} T^{*} M\right)=\delta_{g}^{*} C^{k+1, \alpha}\left(\Lambda^{1}\right) \oplus K
$$

We use a neighbourhood $\mathcal{S}$ of $g$ in $K$ as a slice for the $\mathcal{D}$-action.

Remark 3.7. This is not exactly the same choice of slice as in [2]. It has been used before by Biquard [3] and Kovalev [1].

Let $\mathcal{Q}$ be the space of Ricci-flat (not a priori smooth) metrics in $\mathcal{S}$ - this is the pre-moduli space of Ricci-flat metrics near $g$. The linearisation of the Ricci curvature functional at a Ricci-flat metric is given by (cf. [2, Equation $\left.\left(12.28^{\prime}\right)\right]$ )

$$
(\text { DRic })_{g} h=\triangle_{L} h+\delta_{g}^{*}\left(2 \delta_{g}+d \operatorname{tr}_{g}\right) h,
$$

where $\triangle_{L}$ denotes the Lichnerowicz Laplacian on $S^{2} T^{*} M$ in the sense of definition 2.4 In particular, on the tangent space $K$ to the slice the linearisation reduces to $\triangle_{L}$. This is elliptic so its kernel has finite dimension. Moreover, the kernel of $\triangle_{L}$ is contained in $K$ : differentiating the Bianchi identity (7) at the Ricci-flat metric $g$ gives

$$
\left(2 \delta_{g}+d \operatorname{tr}_{g}\right)(\text { DRic })_{g}=0,
$$

and hence

$$
\triangle_{L} h=0 \Rightarrow \triangle\left(2 \delta_{g}+d \operatorname{tr}_{g}\right) h=0 \Rightarrow\left(2 \delta_{g}+d \operatorname{tr}_{g}\right) h=0 .
$$

Definition 3.8. The space of infinitesimal Ricci-flat deformations of $g$ is the kernel $\varepsilon(g)$ of $\triangle_{L}$ in $\Gamma\left(S^{2}\left(T^{*} M\right)\right)$.

If $h \in \Gamma\left(S^{2} T^{*} M\right)$ is tangent to a curve of Ricci-flat metrics in the slice $\mathcal{S}$ then of course $h \in \varepsilon(g)$. The converse is not true; in general there may be elements in $\varepsilon(g)$ which are not tangent to any curve of Ricci-flat metrics. Thus $\mathcal{Q}$ need not be a manifold with tangent space $\varepsilon(g)$.

The image of $D R i c_{g}$ is the $L^{2}$-orthogonal complement $K^{\prime}$ to $\varepsilon(g)$ in $K$. Let $P_{g}$ be the $L^{2}$-orthogonal projection to $K^{\prime}$. The Ricci curvature functional is real analytic. We can apply the implicit function theorem to the composition

$$
F: \mathcal{S} \rightarrow K^{\prime}: \quad h \mapsto P_{g} \operatorname{Ric}(h)
$$

to deduce that there is a real analytic submanifold $Z \subseteq \mathcal{S}$ whose tangent space at $g$ is precisely $\varepsilon(g)$ and which contains $\mathcal{Q}$ as a real analytic subset. The analyticity implies that if every element of $\varepsilon(g)$ is tangent to a curve of Ricci-flat metrics then in fact $\mathcal{Q}$ contains a neighbourhood of $g$ in $Z$. Thus the pre-moduli space $\mathcal{Q}$ is a manifold in this case (cf. [10, Corollary 3.5]). 
Note that since $K$ is invariant under the isometry group $\mathcal{I}_{g}$ of $g$ we may take $\mathcal{S}, Z$ and $\mathcal{Q}$ to be invariant too. An analogue of proposition 3.2 holds.

Proposition 3.9. For any $g^{\prime} \in \mathcal{Q}$ sufficiently close to $g, \mathcal{I}_{g^{\prime}} \subseteq \mathcal{I}_{g}$. Moreover, the identity components of $\mathcal{I}_{g^{\prime}}$ and $\mathcal{I}_{g}$ are equal.

Proof. The inclusion $\mathcal{I}_{g^{\prime}} \subseteq \mathcal{I}_{g}$ follows from [6, Theorem 7.1(2)]. Proposition 3.6 implies that the dimension of the isometry groups of Ricci-flat metrics is $b^{1}(M)$, so if $\mathcal{I}_{g^{\prime}} \subseteq \mathcal{I}_{g}$ then the identity components must be equal.

The elements of $Z$ are smooth by elliptic regularity (since the linear part of the equation $F(h)=0$ defining $Z$ is $\triangle_{L} h=0$ ), and when $\mathcal{Q}=Z$ it is relatively straight-forward to deduce from the submersion theorem that $\mathcal{Q} \rightarrow \mathcal{W}_{0}$ is open. In general one needs to do a little bit of extra work.

Theorem 3.10. Let $M$ be a compact manifold and $g$ a Ricci-flat metric on $M$. Let $\mathcal{Q}$ be the pre-moduli space of Ricci-flat metrics near $g$, and $\mathcal{I}_{g}$ the stabiliser of $g$ in $\mathcal{D}$. Then $\mathcal{Q} / \mathcal{I}_{g}$ is homeomorphic to a neighbourhood of $g \mathcal{D}$ in $\mathcal{W}_{0}$. In particular, if every element of $\varepsilon(g)$ is integrable then $\mathcal{W}_{0}$ is an orbifold near $g \mathcal{D}$.

Proof. We wish to extend (9) to a function on a neighbourhood $U$ of $g$ in $C^{k, \alpha}\left(S^{2} T^{*} M\right)$ such that $F^{-1}(0)$ is a manifold containing the Ricci-flat metrics in $U$ and ensure that $Z \mathcal{D}_{k+1} \cap U \subseteq$ $F^{-1}(0)$. Then we apply the submersion theorem to deduce that $Z$ contains representatives for all diffeomorphism classes in $F^{-1}(0)$ close to $g$.

By the inverse function theorem, any element of a small neighbourhood $U$ of $g$ can be written as $k+\phi^{*} g^{\prime}$, with $k \in K^{\prime}, \phi \in \mathcal{D}_{k+1}$ and $g^{\prime} \in Z$. Using proposition 3.9.

$$
f: U \rightarrow g \mathcal{D}_{k+1}, \quad k+\phi^{*} g^{\prime} \mapsto \phi^{*} g
$$

is a well-defined smooth function. If $f(h)=\phi^{*} g$ then $P_{f(h)}$ is a projection to $\phi^{*} K^{\prime}$, and we can take

$$
F: U \rightarrow K^{\prime}, h \mapsto P_{g} P_{f(h)} \operatorname{Ric}(h) .
$$

Then $D F_{g}$ maps $K^{\prime}$ onto itself, so $F^{-1}(0)$ is a submanifold of $U$ by the implicit function theorem. By construction it contains both the Ricci-flat metrics in $U$ and $Z \mathcal{D}_{k+1} \cap U$. Now

$$
Z \times \mathcal{D}_{k+1} \rightarrow F^{-1}(0)
$$

is an open map near $(g, i d)$ by the submersion theorem (it is smooth because elements of $Z$ are). This implies that any smooth Ricci-flat metric $g^{\prime}$ near $g$ is $\mathcal{D}_{k+1}$-equivalent to an element of $Z$, which must in fact lie in $\mathcal{Q}$ because Ricci-flatness is a diffeomorphism-invariant property. Since isometries between smooth Riemannian metrics are smooth (see Myers and Steenrod [15, Theorem 8]), $g^{\prime}$ is in fact $\mathcal{D}$-equivalent to an element of $\mathcal{Q}$. In other words, $\mathcal{Q} \rightarrow \mathcal{W}_{0}$ is open.

Proposition 3.9 implies that in fact $\mathcal{Q} \rightarrow \mathcal{W}_{0}$ is injective up to the action of the stabiliser $\mathcal{I}_{g}$ and, since $\mathcal{I}_{g}$ is compact, that the action on $\mathcal{Q}$ factors through a finite group (cf. [2, 12.25]).

Remark 3.11. Clearly the argument would give the same result even if we were to consider the moduli space of Ricci-flat metrics given by dividing by the action of the full diffeomorphism group of $M$.

Remark 3.12. In [10, Lemma 2.6] Koiso uses instead of $\mathcal{S}$ a slice constructed by Ebin [6, and shows that any Einstein metric in this slice is smooth.

3.4. Proof of theorem I. Let $G$ be one of the Ricci-flat holonomy groups, $M$ a compact $G$-manifold, $\Gamma\left(\Lambda_{G} T^{*} M\right)$ the space of $G$-structures on $M$ and

$$
m: \Gamma\left(\Lambda_{G} T^{*} M\right) \rightarrow \Gamma\left(S^{2} T^{*} M\right), \quad \chi \mapsto g_{\chi}
$$

the natural map that sends a $G$-structure to the metric it defines. In order to prove theorem I we show first that for any torsion-free $G$-structure $\chi$ the derivative of $m$ maps the tangent space to the pre-moduli space $\mathcal{R}$ at $\chi$ onto the space $\varepsilon\left(g_{\chi}\right)$ of infinitesimal Ricci-flat deformations.

The tangent space to $\Gamma\left(\Lambda_{G} T^{*} M\right)$ at $\chi$ is the space of differential forms $\Gamma\left(E_{\chi}\right)$, where $E_{\chi} \subseteq$ $\Lambda^{*} T^{*} M$ is a vector subbundle associated to the $G$-structure defined by $\chi$. Fibre-wise $\Lambda_{G} T^{*} M$ is 
a $G L\left(\mathbb{R}^{n}\right)$-orbit and $E_{\chi}$ is the tangent space $\mathfrak{g l}_{n} \chi$ to the orbit. Because $m$ is $G L\left(\mathbb{R}^{n}\right)$-equivariant its derivative takes $a \chi \mapsto a g_{\chi}$ for any $a \in \mathfrak{g l}_{n}$, which maps onto the fibre of $S^{2} T^{*} M$. Hence the derivative

$$
D m_{\chi}: \Gamma\left(E_{\chi}\right) \rightarrow \Gamma\left(S^{2} T^{*} M\right)
$$

is surjective. Furthermore, the derivative is $G$-equivariant with respect to the $G$-structure defined by $\chi$. Since $\triangle_{L}$ is the Lichnerowicz Laplacian on $S^{2} T^{*} M$, lemma 2.6 implies that the diagram below commutes.

Hence

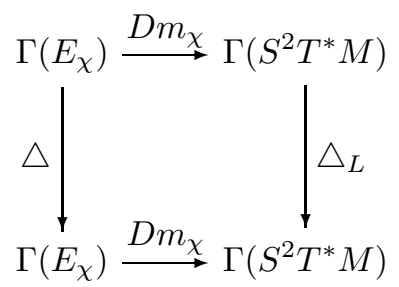

Lemma 3.13. If $\chi$ is a torsion-free $G$-structure then $D m_{\chi}$ maps the harmonic sections of $E_{\chi}$ onto the space $\varepsilon\left(g_{\chi}\right)$ of infinitesimal Ricci-flat deformations.

So let $\chi$ be any torsion-free $G$-structure on $M$ and $\mathcal{R}$ the pre-moduli space of torsion-free $G$-structures near $\chi$. As described in subsection 3.3 there is a slice at $g_{\chi}$ for the $\mathcal{D}$-action on the metrics, the Ricci-flat metrics in the slice are a real analytic subset of a submanifold $Z$, and the tangent space to $Z$ at $\chi$ is $\varepsilon\left(g_{\chi}\right)$. Let $P: F^{-1}(0) \rightarrow Z$ be the composition of a smooth local right inverse to the submersion (11) with the projection to the first factor. $F^{-1}(0)$ contains the Ricci-flat metrics near $g_{\chi}$, and $P$ can be viewed as a local projection to the slice: $P\left(g^{\prime}\right)$ is $\mathcal{D}$-equivalent to $g^{\prime}$ for any Ricci-flat $g^{\prime}$ close to $g_{\chi}$. Then

$$
P \circ m: \mathcal{R} \rightarrow Z
$$

is a well-defined smooth map and lemma 3.13 means that its derivative at $\chi$ is surjective. Therefore every element of $\varepsilon\left(g_{\chi}\right)$ is tangent to a path of Ricci-flat metrics, so $\mathcal{Q}$ is a manifold. By the submersion theorem, $\mathcal{W}_{G}$ (the image of $\mathcal{M}_{G}$ in $\mathcal{W}_{0}$ ) contains a neighbourhood of $g \mathcal{D}$.

The pre-images of $g_{\chi}$ under $m$ are defined by differential forms which are harmonic with respect to $g_{\chi}$. By Hodge theory they represent distinct cohomology classes. Let $\mathcal{I}_{g_{\chi}} \subseteq \mathcal{D}$ be the isometries of $g_{\chi}$ isotopic to the identity. Because $\mathcal{I}_{g_{\chi}}$ acts trivially on cohomology it must fix the fibre over of $m$ over $g_{\chi}$, so $\mathcal{I}_{g_{\chi}}=\mathcal{I}_{\chi}$. Now, if $g^{\prime} \in \mathcal{Q}$ then $g^{\prime}=\phi^{*} m\left(\chi^{\prime}\right)$ for some $\chi^{\prime} \in \mathcal{R}$ and $\phi \in \mathcal{D}_{k+1}$ because (14) is a submersion. As $\mathcal{I}_{\chi}$ acts trivially on $\mathcal{R}$ by proposition 3.2 it follows that the conjugate $\mathcal{I}_{g_{\chi}}^{\phi}$ fixes $g^{\prime}$. But then $\mathcal{I}_{g_{\chi}}^{\phi} \subseteq \mathcal{I}_{g^{\prime}} \subseteq \mathcal{I}_{g_{\chi}}$ by proposition 3.9 so in fact $\mathcal{I}_{g_{\chi}}^{\phi}=\mathcal{I}_{g_{\chi}}$. Hence $\mathcal{I}_{g_{\chi}}$ fixes any $g^{\prime} \in \mathcal{Q}$.

Now theorem 3.10 implies that $\mathcal{Q}$ is homeomorphic to a neighbourhood of $\mathcal{W}_{0}$. Thus $\mathcal{W}_{G}$ is a manifold near $g \mathcal{D}$ and the proof of theorem $\mathrm{I}$ is complete.

3.5. The asymptotically cylindrical case. The proof of theorem I only used the compactness assumption to access certain deformation results for $G$-structures and Ricci-flat metrics. For the cases $G=G_{2}$ and $\operatorname{Spin}(7)$ there are pre-moduli spaces of EAC $G$-structures, with properties analogous to proposition 3.1

If $M$ is an EAC $G$-manifold, let $\mathcal{M}_{G}$ denote the quotient of the space of torsion-free EAC $G$-structures on $M$ by the group $\mathcal{D}$ of EAC diffeomorphisms of $M$ isotopic to the identity.

Proposition 3.14. Let $G=S \sin (7)$ or $G_{2}, M$ an EAC $G$-manifold and $\chi$ a torsion-free EAC $G$-structure on $M$. Then there is a submanifold $\mathcal{R}$ of the space of $C^{1} G$-structures such that

(i) the elements of $\mathcal{R}$ are smooth EAC torsion-free $G$-structures,

(ii) the tangent space to $\mathcal{R}$ at $\chi$ is the space of bounded harmonic sections of $E_{\chi}$,

(iii) the natural map $\mathcal{R} \rightarrow \mathcal{M}_{G}$ is a homeomorphism onto a neighbourhood of $\chi \mathcal{D}$ in $\mathcal{M}_{G}$.

Proof. See [17, §6] for the $G_{2}$ case, and [16, §4.3] for the $\operatorname{Spin}(7)$ case. 
In order to prove the theorem $\mathrm{I}^{\prime}$, the EAC version of theorem I, it therefore suffices to explain how to set up the deformation theory for EAC Ricci-flat metrics. Below we define the slices with same equations as in the compact case in 3.3 and use the same reasoning as for deformations of EAC $G_{2}$-manifolds in [17, §6.7] to make the slice arguments work on EAC manifolds. The resulting approach is similar to that of Kovalev [11, who considers Ricci-flat deformations of EAC Calabi-Yau manifolds.

Let $M^{n}$ be a manifold with cylindrical ends and cross-section $X^{n-1}$. Let $\mathcal{W}_{0}$ be the quotient of the space of EAC Ricci-flat metrics (with any exponential rate) by the group $\mathcal{D}$ of EAC diffeomorphisms of $M$ isotopic to the identity. We pick an EAC Ricci-flat metric $g$ on $M$ and study a neighbourhood of $g \mathcal{D}$ in $\mathcal{W}_{0}$. By definition, the asymptotic limit of $g$ is a cylindrical metric $d t^{2}+g_{X}$ on $X \times \mathbb{R}$, where $g_{X}$ is a Ricci-flat metric on $X$.

We work with weighted Hölder spaces of sections. Let $k \geq 2, \alpha \in(0,1)$, and $\delta>0$ be less than the exponential rate of $g$. The metric $g$ defines a Hodge Laplacian on 1-forms and a Lichnerowicz Laplacian on symmetric bilinear forms, which are both asymptotically translation-invariant operators. We require that $\delta$ is small enough that the Laplacians are Fredholm on $C_{\delta}^{k, \alpha}$ spaces, as we may according to theorem 2.8 .

We proved in $\$ 3.3$ that there is a real analytic submanifold $Z \subset C^{k, \alpha}\left(S^{2} T^{*} X\right)$ which contains representatives of all diffeomorphism classes of Ricci-flat metrics on $X$ close to $g_{X}$. Its tangent space $T_{g_{X}} Z=\varepsilon\left(g_{X}\right)$ is the space of Lichnerowicz harmonic sections of $S^{2} T^{*} X$.

Let $\mathscr{M}_{Z}^{k}$ denote the space of $C^{k, \alpha}$ metrics on $M$ which are $C_{\delta}^{k, \alpha}$-asymptotic to cylindrical metrics $d t+g_{X}^{2}$ such that $g_{X} \in Z$. If $\rho$ is a cut-off function for the cylinder then $\rho Z$ can be identified with a space of bilinear forms on $M$, and $\mathscr{M}_{Z}^{k}$ is an open subset

$$
\mathscr{M}_{Z}^{k} \subset C_{\delta}^{k, \alpha}\left(S^{*} T^{*} M\right)+\rho Z .
$$

Similarly let $\mathcal{D}_{Z}^{k+1}$ be the set of EAC diffeomorphisms with rate $\delta$ which are asymptotic to elements of the isometry group $\mathcal{I}_{g_{X}}$ of $g_{X}$. Then $\mathscr{M}_{Z}^{k}$ contains representatives of all diffeomorphism classes of Ricci-flat metrics near $g$ and, because $Z$ is $\mathcal{I}_{g_{X}}$-invariant, proposition 3.9 implies that any isometry between elements of $\mathscr{M}_{Z}^{k}$ must lie in $\mathcal{D}_{Z}^{k+1}$ (a similar argument for simplifying the problem by a slice at the boundary was used to study the moduli space of torsion-free EAC $G_{2}$-structures in [17, Lemma 6.24]). We therefore identify a slice in $\mathscr{M}_{Z}^{k}$ for the action of $\mathcal{D}_{Z}^{k+1}$ at $g$. The tangent space to $\mathscr{M}_{Z}^{k}$ at $g$ is

$$
T_{g} \mathscr{M}_{Z}^{k}=C_{\delta}^{k, \alpha}\left(S^{*} T^{*} M\right) \oplus \rho \varepsilon\left(g_{X}\right) .
$$

The tangent space at the identity of $\mathcal{D}_{Z}^{k+1}$ corresponds to vector fields which are $C_{\delta}^{k, \alpha}$-asymptotic to translation-invariant Killing vector fields on the cylinder, i.e. to elements of $\left(\mathcal{H}_{\infty}^{1}\right)^{\sharp}$, where $\mathcal{H}_{\infty}^{1}$ denotes the translation-invariant harmonic 1-forms on the cylinder $X \times \mathbb{R}$. By proposition 3.4 the tangent space to the $\mathcal{D}_{Z}^{k+1}$-orbit at $g$ is

$$
\delta_{g}^{*}\left(C_{\delta}^{k, \alpha}\left(\Lambda^{1}\right) \oplus \rho \mathcal{H}_{\infty}^{1}\right)
$$

Let $K$ be the kernel of $2 \delta_{g}+d \operatorname{tr}_{g}$ in $T_{g} \mathscr{M}_{Z}^{k}$.

Lemma 3.15. Let $M$ be a Ricci-flat EAC manifold with a single end. Then

$$
T_{g} \mathscr{M}_{Z}^{k}=K \oplus \delta_{g}^{*}\left(C_{\delta}^{k, \alpha}\left(\Lambda^{1}\right) \oplus \rho \mathcal{H}_{\infty}^{1}\right) .
$$

Proof. $\left(2 \delta_{g}+d \operatorname{tr}_{g}\right) \delta^{*}=\triangle_{g}$ according to lemma 3.5 so it suffices to show that the image of $2 \delta_{g}+d \operatorname{tr}_{g}: T_{g} \mathscr{M}_{Z}^{k} \rightarrow C_{\delta}^{k-1, \alpha}\left(\Lambda^{1}\right)$ is contained in the image of

$$
\triangle: C_{\delta}^{k+1, \alpha}\left(\Lambda^{1}\right) \oplus \rho \mathcal{H}_{\infty}^{1} \rightarrow C_{\delta}^{k-1, \alpha}\left(\Lambda^{1}\right) .
$$

It follows from theorem 2.8 that this has index 0 , so its image is the $L^{2}$-orthogonal complement to its kernel $\mathcal{H}_{0}^{1}$, the space of bounded harmonic 1 -forms.

Now, if $h \in T_{g} \mathscr{M}_{Z}^{k}$ and $\beta \in \mathcal{H}_{0}^{1}$ then the difference between $\left\langle\delta_{g} h, \beta>\right.$ and $\left\langle h, \delta_{g}^{*} \beta\right\rangle=0$ is the divergence of the contraction of $h$ with $\beta$. The boundary condition on $h$ ensures that the asymptotic limit of the contraction has no $d t$-component, so the integral of the divergence is 0 . Hence

$$
<\delta_{g} h, \beta>_{L^{2}}=0 .
$$


The hypothesis that $M$ has a single end ensures that the asymptotic limit of $\beta$ has no $d t$-component (corollary 2.10), so integration by parts also applies to show that $\left.<d \operatorname{tr}_{g} h, \beta\right\rangle_{L^{2}}=0$. Thus the image of $2 \delta_{g}+d \operatorname{tr}_{g}$ is $L^{2}$-orthogonal to $\mathcal{H}_{0}^{1}$.

Now we can use a real analytic $\mathcal{I}_{g}$-invariant submanifold $\mathcal{S} \subset \mathscr{M}_{Z}^{k}$ with $T_{g} \mathcal{S}=K$ as a slice for the $\mathcal{D}_{Z}^{k+1}$-action. Let $\mathcal{Q} \subset \mathcal{S}$ be the subset of Ricci-flat metrics. As in the compact case $\mathcal{Q}$ is an analytic subset of an analytic submanifold $Z^{\prime} \subset \mathcal{S}$, defined as the zero set of the composition of the Ricci functional $\mathcal{S} \rightarrow C_{\delta}^{k-2, \alpha}\left(S^{2} T^{*} M\right)$ with the projection onto the image of its derivative at $g$. On $K$ the derivative of the Ricci functional is the Lichnerowicz Laplacian, so $T_{g} Z^{\prime}$ is the space of harmonic sections of $S^{2} T^{*} M$, exponentially asymptotic to sections of $S^{2} T^{*} X$ (i.e. the asymptotic limit has no $d t$-components). This is the space of infinitesimal Ricci-flat EAC deformations $\varepsilon(g)$.

In general we can use regularity and arguments like in [17, §6.6] to show that $Z$ consists of smooth EAC metrics, and then extend the proof of theorem 3.10 , thus proving that $\mathcal{Q} / \mathcal{I}_{g}$ is homeomorphic to a neighbourhood of $g$ in $\mathcal{W}_{0}$.

We have now set up the deformation theory for EAC Ricci-flat metrics that is required, together with the unobstructedness of deformations of torsion-free EAC $G$-structures for $G=G_{2}$ and $\operatorname{Spin}(7)$ stated in proposition 3.14, in order to prove theorem I' by the same argument as for the compact case.

\section{REFERENCES}

[1] M. Berger. Sur les groupes d'holonomie homogène des variétés à connexion affines et des variétés riemanniennes. Bull. Soc. Math. France, 83:279-330, 1955.

[2] A.L. Besse. Einstein Manifolds. Springer-Verlag, New York, 1987.

[3] O. Biquard. Métriques d'Einstein asymptotiquement symétriques. Astérisque, 265, 2000.

[4] S.S. Chern. On a generalization of Kähler geometry. In Algebraic Geometry and Topology, A Symposium in honor of S. Lefschetz, pages 103-121. Princeton University Press, 1957.

[5] X. Dai, X. Wang, and G. Wei. On the stability of Riemannian manifold with parallel spinors. Invent. Math., 161:151-176, 2005.

[6] D.G. Ebin. The manifold of Riemannian metrics. In Global Analysis, volume 15 of Proc. Symp. Pure. Math., pages 11-40. Amer. Math. Soc., Providence, 1970.

[7] R. Goto. Moduli spaces of topological calibrations, Calabi-Yau, hyperKähler, $G_{2}$ and $\operatorname{Spin}(7)$ structures. Internat. J. Math., 115(3):211-257, 2004.

[8] N.J. Hitchin. The moduli space of special Lagrangian submanifolds. Ann. Scuola Sup. Norm. Pisa Sci. Fis. Mat., 25:503-515, 1997.

[9] D.D. Joyce. Compact Manifolds with Special Holonomy. OUP Mathematical Monographs series. Oxford University Press, 2000.

[10] N. Koiso. Einstein metrics and complex structures. Invent. Math., 73:71-106, 1983.

[11] A.G. Kovalev. Ricci-flat deformations of asymptotically cylindrical Calabi-Yau manifolds. In Proceedings of Gökova Geometry-Topology Conference 2005, pages 140-156. International Press, 2006.

[12] R.B. Lockhart. Fredholm, Hodge and Liouville theorems on noncompact manifolds. Trans. Amer. Math. Soc., 301:1-35, 1987.

[13] R.B. Lockhart and R.C. McOwen. Elliptic differential operators on noncompact manifolds. Ann. Scuola Norm. Sup. Pisa Cl. Sci., 12:409-447, 1985.

[14] R. Melrose. The Atiyah-Patodi-Singer index theorem. AK Peters, Wellesley, MA, 1994.

[15] S.B. Myers and N.E. Steenrod. The group of isometries of a riemannian manifold. Ann. Math., 40:400-416, 1939.

[16] J. Nordström. Deformations and gluing of asymptotically cylindrical manifolds with exceptional holonomy. $\mathrm{PhD}$ thesis, University of Cambridge, 2008.

[17] J. Nordström. Deformations of asymptotically cylindrical G2-manifolds. Math. Proc. Camb. Phil. Soc., 145:311-348, 2008.

[18] S.M. Salamon. Riemannian Geometry and Holonomy Groups, volume 201 of Pitman Reseach Notes in Mathematics. Longman, Harlow, 1989.

[19] G. Tian. Smoothness of the universal deformation space of compact Calabi-Yau manifolds and its PeterssonWeil metric. In Math. Aspects of String Theory, pages 629-646, 1987.

[20] A.N. Todorov. The Weil-Petersson geometry of the moduli space of $S U(n \geq 3)$ (Calabi-Yau) manifolds, I. Comm. Math. Phys., 126:325-346, 1989.

[21] M.Y. Wang. Parallel spinors and parallel forms. Ann. Global Anal. Geom., 7:59-68, 1989.

[22] M.Y. Wang. Preserving parallel spinors under metric deformations. Indiana Univ. Math. J., 40:815-844, 1991.

Department of Mathematics, Imperial College London, London SW7 2AZ, UK

E-mail address: j.nordstrom@imperial.ac.uk 